\title{
Determinación en Terreno de la Transmisibilidad de Asientos en Grúas de Horquilla y su Efecto en la Exposición Ocupacional a Vibración de Cuerpo Entero
}

\author{
DETERMINATION IN FIELD OF TRANSMISSIBILITY OF SEATS FORKLIFT TRUCKS AND THEIR EFFECT ON OCCU- \\ PATIONAL EXPOSURE TO WHOLE BODY VIBRATION
}

\author{
Alonso Carrillo Mayorga ${ }^{1}$, Rómulo Zúñiga $\mathrm{R}^{2}$ \\ 1. Ingeniero Acústico, Diplomado en Ergonomía, Máster en Higiene Industrial. \\ 2. Ingeniero Civil Químico.
}

\begin{abstract}
RESUMEN
Se estudió el aislamiento a la vibración, por medio del factor SEAT (Seat Effective Amplitude Transmissibility), de 4 tipos de asientos de grúa de horquilla, los cuales se seleccionaron por poseer distintos sistemas de amortiguamiento a las vibraciones.

Los asiento utilizados fueron los modelos GRAMMER MSG71GBLV (SEAT $=0,55$ en el eje Z), KAB Seating 21/T1 (SEAT $=0,96$ en el eje Z), GENÉRICO BF2-3 (SEAT = 1,01 en el eje Z) y GENÉRICO BFL-3 (SEAT $=0,82$ en el eje Z).

Para obtener los valores SEAT, se efectuaron mediciones de 30 minutos para 2 condiciones, desplazamiento del vehículo sin carga durante 20 minutos por una ruta de 6 kilómetros y, luego, simulando una condición de trabajo habitual de una grúa de horquilla para trabajo cíclico con carga por un período de tiempo aproximado de 10 minutos.

Adicionalmente, se evaluó la exposición a vibración del operador de la grúa de horquilla seleccionada según el D.S. $N^{\circ}$ 594/1999 y Directiva 2002/44/CE, documentos basados en la norma ISO 2631-1, con el fin de estudiar el efecto de los asientos en la exposición del operador y el nivel de riesgo asociado, para entregar recomendaciones sobre la selección y uso de asientos en grúas de horquilla.
\end{abstract}

(Carrillo A, Zúñiga R, 2014. Determinación en Terreno de la Transmisibilidad de Asientos en Grúas de Horquilla y su Efecto en la Exposición Ocupacional a Vibración de Cuerpo Entero. Cienc Trab. MayAgo; 16 [50]: 75-80).

Palabras claves: VIBRACIÓN DE CUERPO ENTERO, EXPOSICIÓN A VIBRACIÓN, ISO2631-1, VIBRACIÓN EN GRÚAS DE HORQUILLA, SEAT.

\section{ABSTRACT}

It was studied the isolation of vibration by means of factor SEAT (Seat Effective Amplitude transmissibility) on 4 types of seat forklift truck, which were selected for having different systems of vibration damping. The seat models used were GRAMMER MSG71GBLV (SEAT $=0.55$ in the $Z$ axis), KAB Seating 21/T1 (SEAT $=0.96$ in the $Z$ axis), Generic $\mathrm{BFL}-3(\mathrm{SEAT}=1.01$ in the axis $\mathrm{Z})$ and generic BFL-3 $(\mathrm{SEAT}=0.82 \mathrm{in}$ the $\mathrm{Z}$ axis).

To obtain SEAT values, it were performed measurements of 30 minutes for 2 conditions, moving vehicle without load for 20 minutes by a 6 kilometer route and, then, simulating a typical work of a forklift truck to cyclic work with load for a period of time of approximately 10 minutes. Additionally, the exposure to vibration of selected forklift truck operator was evaluated according to the DS N ${ }^{\circ} 594 / 1999$ and Directive 2002/44/EC documents based on ISO 2631-1, in order to study the effect of the seats in operator exposure and the level of associated risk, to provide recommendations on selection and use of forklift trucks seats.

Key words: WHOLE BODY VIBRATION, VIBRATION EXPOSURE, ISO2631-1, VIBRATION IN FORKLIFTS TRUCKS, SEAT.

\section{INTRODUCCIÓN}

Las grúas de horquillas son vehículos versátiles, destinados al desarrollo de tareas de carga, traslado y descarga de materiales. Para levantar una carga, estos vehículos portan un contrapeso en la parte posterior proporcional a su máxima capacidad de carga.

Correspondencia / Correspondence:

Alonso Carrillo Mayorga

Rómulo Zúñiga $R$

Ramón Carnicer 1634, Providencia- Chile

Tel.: (02) 25157564

e-mail: rzuniga@achs.cl; alonso.carrillo@outlook.com

Recibido: 09 de Julio 2014 / Aceptado: 19 de Julio 2014
El tren trasero presenta una alta rigidez y bajo amortiguamiento ante las vibraciones, quedando la función de reducción del movimiento solamente a cargo del asiento del operador. Este actuaría a modo de elemento de protección personal si su capacidad de reducción fuese adecuada, lo cual habría que evaluar. Una grúa de horquilla con una alta frecuencia de uso normalmente debe reponer su asiento cada uno o dos años por deterioro.

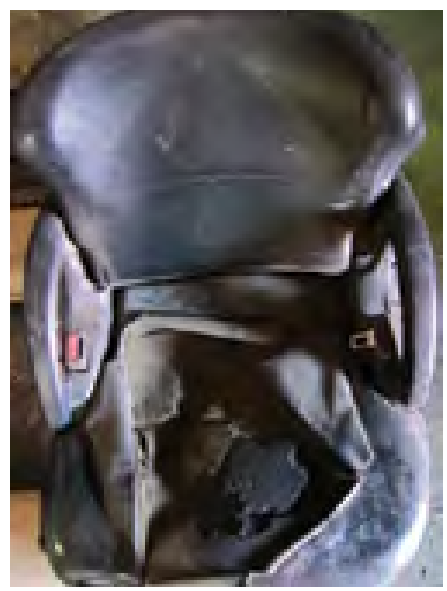




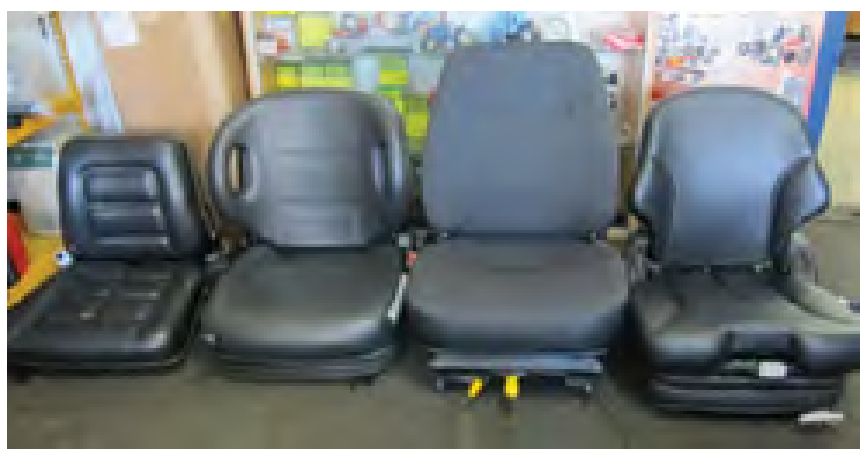

Los operadores de grúas de horquilla están expuestos a vibración mecánica que, cuando se transmite a todo el cuerpo, puede implicar riesgos para la salud y la seguridad; en particular, lumbalgias y lesiones de la columna vertebral. La transmisión de la vibración al cuerpo entero sucede, usualmente, a través de partes de éste, como glúteos, plantas de los pies, espalda, etc., que están en contacto con una superficie que vibra, tal como el asiento, siendo su estudio fundamental en la prevención de las enfermedades laborales asociadas.

La transmisibilidad describe la magnitud a la cual la vibración es transmitida desde un punto de entrada a uno de salida que, en este trabajo, estará determinada por la razón de la vibración de salida para el trabajador, superficie superior del asiento respecto a la vibración de entrada o la que se manifiesta en la base del asiento. La normativa nacional para evaluar la exposición ocupacional de un trabajador a vibración, Decreto Supremo N ${ }^{\circ}$ 594/1999 ${ }^{1}$, establece como índice de medición la aceleración equivalente ponde-

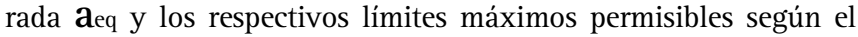
tiempo de exposición para aceleraciones de cuerpo entero. Por otro lado, la normativa internacional ISO 2631-12 establece también el indicador "dosis", que es considerado adecuado para evaluar exposiciones que contengan choques, estableciendo además un límite de acción inferior al límite de exposición utilizado para adoptar acciones preventivas, como la incorporación de una persona expuesta a vibración a un programa de vigilancia de salud.

\section{MATERIAL Y MÉTODOS}

\section{Grúa de Horquilla}

Las mediciones se realizaron en una grúa horquilla marca TOYOTA, modelo 42-6FG20 año 1997, serie $\mathrm{N}^{\circ} 4028$, accionada con gas. Su capacidad de carga es de $2000 \mathrm{~kg}$ con una altura máxima de 4,3 m. Posee dirección hidráulica $\mathrm{y}$ transmisión automática, frenos hidráulicos

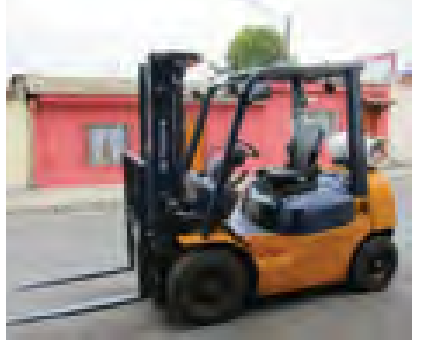
de poder, con neumáticos delanteros inflados 700/12 y neumáticos traseros macizos 600/9.

\section{Asientos}

Los tipos de asientos utilizados en el proyecto fueron:

a) 3 asientos con tecnología reductora de vibraciones marca GRAMMER, Modelo MSG71GBLV, el cual posee un compresor de aire de $12 \mathrm{~V}$ para el control del sistema de suspensión neumático. Se regula con el peso del conductor. b) 3 asientos con tecnología reductora de vibraciones marca $\mathrm{KAB}$ Seating, Modelo 21/T1, con un sistema de suspensión que incorpora un amortiguador hidráulico central y dos resortes laterales. c) 3 asientos convencionales marca Genérico Modelo BF2-3, con un sistema de suspensión mecánica de resorte integrado al cojín. d) 3 asientos convencionales marca Genérico Modelo BFL-3, con un sistema de suspensión por resortes integrados al respaldo.

\section{Instrumentación y Montaje}

Se utilizó un acelerómetro triaxial Larson Davis SEN021 en la base del asiento, asignado a los canales 4, 5 y 6 (Z, Y, X respectivamente) del medidor de vibraciones SVANTEK SV106, mientras que un acelerómetro DYTRAN 3143 M5 montado en un disco de caucho estandarizado se ubicó sobre el asiento de la grúa de horquilla, asignando éste a los canales 1, 2 y 3 (Z, Y, X respectivamente) del medidor (ver Figura 2). El instrumento de medición se fijó en uno de los pilares de la cabina de la grúa de horquilla (ver Figura 1).

Figura 1.

Montaje medidor de vibraciones.

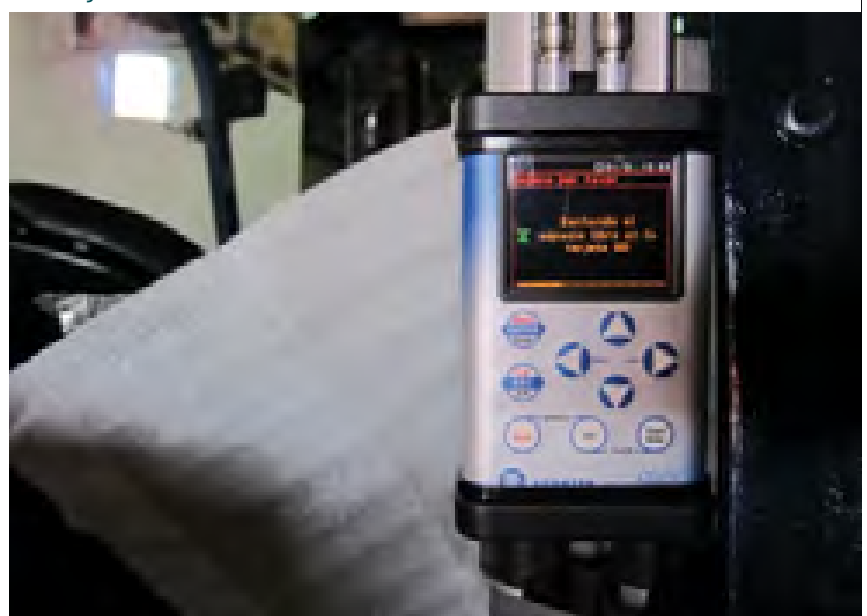

Adicionalmente se usaron dos sensores triaxiales AHRS YEI TECHNOLOGIES modelo 3 SPACE DATALOGGER, con el objeto de registrar la señal de vibración en función del tiempo para cada asiento. Uno de estos sensores se adhirió al disco de caucho, mientras que el otro se adhirió sobre el acelerómetro triaxial Larson Davis SEN021 (Figura 2).

Figura 2.

Montaje de acelerómetros en asiento y su base.

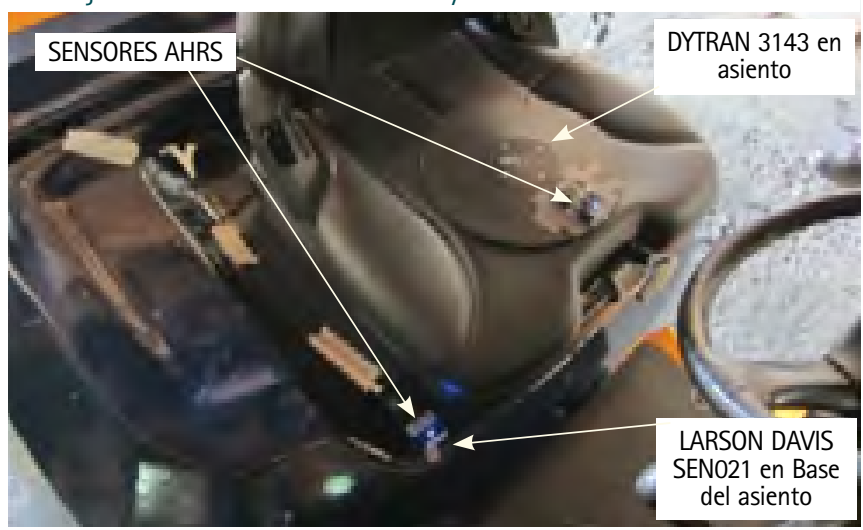




\section{Condiciones de Medición}

Cada medición fue de 30 minutos continuos, separados en dos condiciones: "sin carga" y "con carga".

La primera condición "sin carga” consistió en el desplazamiento de la grúa de horquilla por una ruta determinada por calles de la ciudad de Viña del Mar, con una longitud aproximada de $6 \mathrm{~km}$. Para asegurar que la velocidad de desplazamiento se mantenga constante, se reguló el acelerador del vehículo con un perno utilizado como tope, para alcanzar una velocidad máxima de $20 \mathrm{~km} / \mathrm{h}$, instruyendo al operador de la grúa de horquilla para mantener el acelerador unido al tope señalado.

La segunda condición "con carga" consistió en el trabajo que realiza normal y habitualmente una grúa de horquilla, el desplazamiento con carga en un trayecto reducido, actividad desarrollada en un galpón de la empresa THENOUX Ltda., inmediatamente después de terminar el desplazamiento de 20 minutos en el circuito de $6 \mathrm{Km}$, para lo cual se armó un pallet con un peso aproximado de $300 \mathrm{~kg}$, para que la grúa cargue y descargue con desplazamientos cíclicos reducidos, en un trayecto de aproximadamente $20 \mathrm{~m}$. La medición en esta condición se desarrolló en un tiempo aproximado de 10 minutos por cada asiento.

Metodología para la Determinación de la Transmisibilidad y SEAT Las curvas de transmisibilidad, reducción de vibración en función de la frecuencia, se elaboraron a partir de los espectros de frecuencia RMS en 1/3 de octava obtenidos en los seis canales del medidor de vibraciones SVANTEK SV106, y luego realizando la razón entre el espectro medido en el asiento y el espectro medido en la base, comparando los canales $1 / 4(\mathrm{Z}), 2 / 5(\mathrm{Y})$ y $3 / 6(\mathrm{X})$. Con esto se obtienen 3 curvas de transmisibilidad por tipo de asiento, para luego promediarlas y obtener una curva de transmisibilidad representativa de un tipo de asiento.

El factor SEAT se obtuvo para los tres ejes y para los indicadores RMS y VDV, realizando la razón entre el espectro medido en el asiento y el espectro medido en su base, ponderando las aceleraciones del eje $\mathrm{Z}$ por $\mathrm{W}_{\mathrm{k}} \mathrm{y}$ las de ejes $\mathrm{X}$ e $\mathrm{Y}$ por $\mathrm{W}_{\mathrm{d}}$. Se obtienen 3 SEAT por cada tipo de asiento, promediándose éstos para conseguir un SEAT final representativo por tipo de asiento.

\section{Metodología para la Evaluación de la Exposición}

i. Para simular una condición "típica" de exposición de un operador de grúa de horquilla, primero se determinó un tiempo de exposición total de 6 horas, obtenido de la estadística de mediciones efectuadas en ACHS entre 1999 y 2010 a 86 operadores de grúa de horquilla, con una combinación 70\% de actividad "con carga" y un 30\% "sin carga". La composición de las vibraciones en las dos condiciones de medición y en los porcentajes señalados permitieron obtener el valor de exposición final del operador del vehículo. También se evaluó la exposición para cada condición individual; en suma, planteándose los siguientes casos de exposición para el análisis:

- condición "sin carga” (aprox. $20 \mathrm{~min}$ ).

- condición "con carga” (aprox.10 min).

- condición "sin carga-con carga" ( (a) + (b), aprox. 30 min).

- condición "típica”, 30\% sin carga - 70\% con carga.

\section{RESULTADOS}

\section{Vibración de Entrada en Base del Asiento}

A modo de ilustrar la vibración a la que fue sometido cada tipo de asiento, el Gráfico 1 muestra el espectro de frecuencia promedio de aceleración obtenido de los doce espectros RMS medidos al reproducir la condición "sin carga-con carga".

\section{Gráfico 1.}

Espectro de frecuencia promedio de aceleración en bandas de tercio de octava, condición "sin carga-con carga" para la base del asiento.

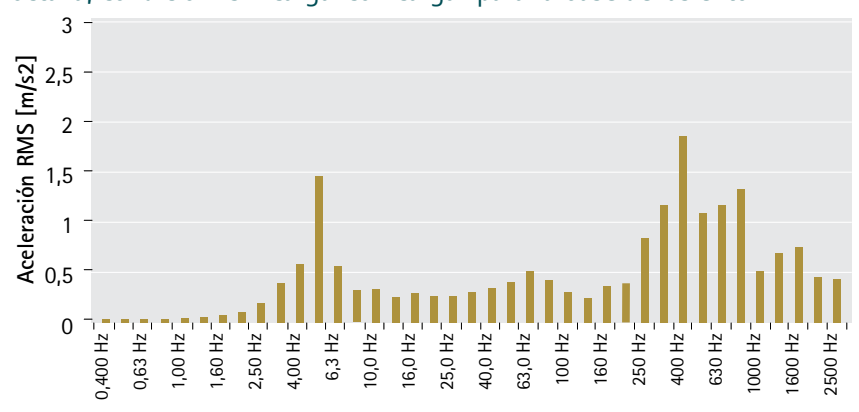

Frecuencia $(\mathrm{Hz})$

\section{Transmisibilidad}

El Gráfico 2 muestra la curva de transmisibilidad promedio por cada tipo de asiento, en el rango de frecuencia entre $1 \mathrm{~Hz}$ a 125 $\mathrm{Hz}$, comparadas con la de un asiento rígido teórico.

\section{Gráfico 2.}

Espectros de transmisibilidad promedio de cada tipo de asiento, comparados con un asiento rígido (teórico). Valores inferiores a 1 implican reducción de vibración.

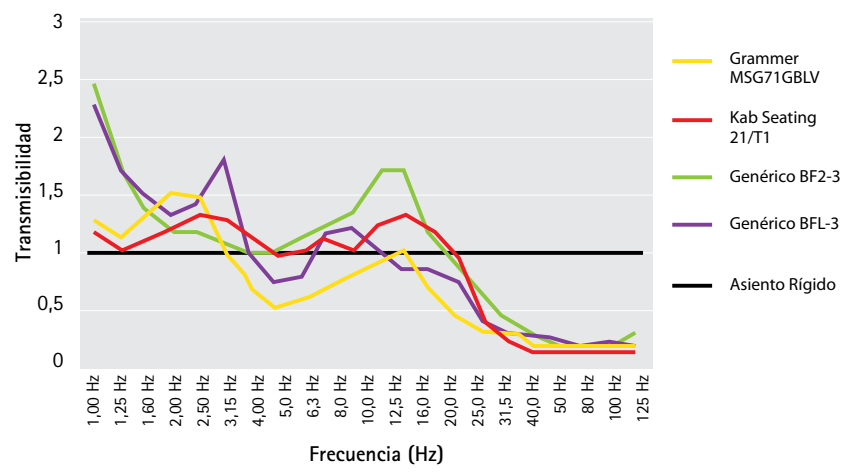

\section{SEAT (Seat Effective Amplitude Transmissibility)}

Los valores SEAT para cada tipo de asiento, comparando los canales $1 / 4(\mathrm{Z}), 2 / 5(\mathrm{Y})$ y $3 / 6(\mathrm{X})$ del instrumento medidor, son los que se presentan en la Tablas 1, 2 y 3 para los ejes Z, Y y X, respectivamente.

Tabla 1.

SEAT RMS y VDV en eje $Z$.

$\begin{array}{lcc}\text { Tipo de Asiento } & \text { SEAT RMS } 1 / 4 \text { Promedio } & \text { SEAT VDV } 1 / 4 \text { Promedio } \\ \text { Grammer MSG71GBLV } & 0,55 & 0,57 \\ \text { KAB Seating 21/T1 } & 0,96 & 0,91 \\ \text { Genérico BF2-3 } & 1,01 & 1,03 \\ \text { Genérico BFL-3 } & 0,82 & 0,80\end{array}$

Tabla 2.

SEAT RMS y VDV en eje $Y$.

$\begin{array}{lcc}\text { Tipo de Asiento } & \text { SEAT RMS } 2 / 5 \text { Promedio } & \text { SEAT VDV 2/5 Promedio } \\ \text { Grammer MSG71GBLV } & 1,28 & 1,34 \\ \text { KAB Seating 21/T1 } & 1,28 & 1,32 \\ \text { Genérico BF2-3 } & 1,19 & 1,18 \\ \text { Genérico BFL-3 } & 1,14 & 1,12\end{array}$


Tabla 3 .

SEAT RMS y VDV en eje X.

$\begin{array}{lcc}\text { Tipo de Asiento } & \text { SEAT RMS 2/5 Promedio } & \text { SEAT VDV 2/5 Promedio } \\ \text { Grammer MSG71GBLV } & 1,30 & 1,25 \\ \text { KAB Seating 21/T1 } & 1,47 & 1,38 \\ \text { Genérico BF2-3 } & 1,74 & 1,66 \\ \text { Genérico BFL-3 } & 1,87 & 1,82\end{array}$

Tabla 4 .

Limites permisibles D.S № 594/1999 para 6 horas de exposición y calificación de la exposición.

$\begin{array}{lccc}\text { EJE Z } & \text { EJEY } & \text { EJE X } & \begin{array}{c}\text { CALIFICACIÓN } \\ \text { EXPOSICIÓN }\end{array} \\ a_{e q}>0,78 \mathrm{~m} / \mathrm{s}^{\wedge} 2 & a_{e q}>0,54 \mathrm{~m} / \mathrm{s}^{\wedge} 2 & a_{e q}>0,54 \mathrm{~m} / \mathrm{s}^{\wedge} 2 & \text { SOBRE EL LIMITE } \\ a_{e q}<0,78 \mathrm{~m} / \mathrm{s}^{\wedge} 2 & a_{e q}<0,54 \mathrm{~m} / \mathrm{s}^{\wedge} 2 & a_{e q}<0,54 \mathrm{~m} / \mathrm{s}^{\wedge} 2 & \text { BAJO EL LIMITE }\end{array}$

\section{Evaluación de la Exposición Según D.S.N594/1999}

En la Tabla 4 se presentan los límites máximos permisibles para un tiempo de exposición de 6 horas, según el D.S. N 594/1999. Adicionalmente se muestra una calificación de la exposición. El resultado de la evaluación de la exposición para las condiciones de exposición planteados se muestran en las Tablas 5, 6, 7 y 8.

Tabla 5 .

Evaluación condición "sin carga" (tiempo de medición aprox. 20 min).

\begin{tabular}{|l|c|c|c|}
\hline Tipo de Asiento & Eje Z $(\mathbf{W k})$ & $\begin{array}{c}a_{e q} \mathrm{~m} / \mathrm{s}^{\wedge} 2 \\
\text { Eje } \mathbf{Y}(\mathrm{Wd})\end{array}$ & Eje X (Wd) \\
\hline Grammer MSG71GBLV & 1,073 & 0,353 & 0,322 \\
\hline KAB Seating 21/T1 & 1,864 & 0,371 & 0,370 \\
\hline Genérico BF2-3 & 2,086 & 0,345 & 0,492 \\
\hline Genérico BFL-3 & 2,094 & 0,331 & 0,525 \\
\hline
\end{tabular}

Tabla 6 .

Evaluación condición "con carga" (tiempo de medición aprox. 10 min).

\begin{tabular}{|l|c|c|c|}
\hline Tipo de Asiento & Eje Z $(W k)$ & $\begin{array}{c}a_{\text {eq }} \mathrm{m} / \mathrm{s}^{\wedge} 2 \\
\text { Eje } Y(W d)\end{array}$ & Eje $X(W d)$ \\
\hline Grammer MSG71GBLV & 0,593 & 0,319 & 0,279 \\
KAB Seating 21/T1 & 1,058 & 0,333 & 0,313 \\
Genérico BF2-3 & 1,103 & 0,310 & 0,411 \\
Genérico BFL-3 & 1,057 & 0,267 & 0,404 \\
\hline
\end{tabular}

Tabla 7 .

Evaluación Exposición "sin carga y con carga" (tiempo de medición aprox. $30 \mathrm{~min}$ ).

\begin{tabular}{|l|c|c|c|}
\hline Tipo de Asiento & Eje Z $(\mathbf{W k})$ & $\begin{array}{c}a_{\text {eq }} \mathrm{m} / \mathrm{s}^{\wedge} 2 \\
\mathrm{Eje} Y(W d)\end{array}$ & Eje X (Wd) \\
\hline Grammer MSG71GBLV & 0,960 & 0,344 & 0,311 \\
\hline KAB Seating 21/T1 & 1,678 & 0,360 & 0,355 \\
\hline Genérico BF2-3 & 1,851 & 0,335 & 0,469 \\
\hline Genérico BFL-3 & 1,915 & 0,317 & 0,497 \\
\hline
\end{tabular}

Tabla 8 .

Evaluación Exposición "Típica", 30\% sin carga-70\% con carga.

\begin{tabular}{|c|c|c|c|}
\hline \multirow[t]{2}{*}{ Tipo de Asiento } & \multicolumn{3}{|c|}{$a_{e q} \mathrm{~m} / \mathrm{s}^{\wedge} 2$} \\
\hline & $Z(W k)$ & $Y(W d)$ & $X(W d)$ \\
\hline Grammer MSG71GBLV & 0,77 & 0,33 & 0,29 \\
\hline KAB Seating 21/T1 & 1,35 & 0,34 & 0,33 \\
\hline Genérico BF2-3 & 1,47 & 0,32 & 0,44 \\
\hline Genérico BFL-3 & 1,45 & 0,29 & 0,44 \\
\hline
\end{tabular}

Evaluación de la Exposición Según Directiva 2002/44/CE

Esta normativa de la Unión Europea, derivada de la norma ISO 2631-1, a diferencia de la normativa chilena cuenta con un límite permisible (ELV) y un límite de acción (EAV), junto con dos indicadores de evaluación, la aceleración RMS ponderada (si el Factor de Cresta es menor o igual a 9) y la dosis de vibración VDV (si el Factor de Cresta es mayor que 9).

La Tabla 9 muestra el criterio de evaluación de la exposición según el factor de cresta de la vibración, en términos de la aceleración RMS ponderada y Dosis (VDV), junto con la calificación del riesgo. Las Tablas 10, 11, 12 y 13 muestran las aceleraciones RMS y valores de dosis VDV resultantes, junto con la calificación de la exposición para las distintas condiciones de evaluación propuestas.

Tabla 9 .

Criterio de Evaluación y Calificación de la Exposición según Nivel de Riesgo, directiva 2002/44/CE.

$\begin{array}{ccc}\text { Criterio de Evaluación si } & \text { Criterio de Evaluación si } & \\ \text { Factor de Cresta } \leq 9 & \text { Factor de Cresta }>9 & \text { Calificación Riesgo } \\ \text { aeq(8) }>\text { ELV } & \operatorname{VDV}(8)>\text { ELV } & \text { CRíTICO } \\ \text { EAV }<\text { aeq(8) }<\text { ELV } & \text { EAV }<\operatorname{VDV}(8)<\text { ELV } & \text { IMPORTANTE } \\ \text { aeq(8) }<\text { EAV } & \operatorname{VDV}(8)<\text { EAV } & \text { BAJO }\end{array}$

\section{CONCLUSIONES}

\section{Asiento GRAMMER MSG71GBLV}

Resultó con un SEAT promedio en el eje $\mathrm{Z}$ de 0,55 (RMS) y 0,57 (VDV) que indica una reducción de 45\% y 43\% en las vibraciones. Fue el único asiento que produjo una reducción de las vibraciones a un nivel tal que la exposición resultó bajo el límite permisible del D.S. No 594/1999 ${ }^{1}$ en la condición "con carga” y simulando la condición "típica” de trabajo de 30\% sin carga y 70\% con carga. Esto se atribuye a su sistema de suspensión neumático que logra reducir la vibración a partir de una frecuencia de corte de $3,5 \mathrm{~Hz}$, inferior a la frecuencia de resonancia del sistema de suspensión de la grúa de horquilla, en torno a $\operatorname{los} 5 \mathrm{~Hz}$, pudiendo reducir esta componente vibratoria en un porcentaje cercano al $43 \%$ y con porcentajes mayores para las componentes de frecuencia superiores. Los valores SEAT en X e Y fueron superiores al valor 1, pero las vibraciones en estos ejes son menos significativas que las del eje $\mathrm{Z}$ en la exposición del operador.

\section{Asiento KAB SEATING 21/T1}

En el eje Z, resultó con un SEAT promedio de 0,96 (RMS) y 0,91 (VDV) que indica una reducción de 4\% y 9\% en las vibraciones. En el mismo eje, en todas las condiciones de exposición estudiadas, se sobrepasa el límite permisible del D.S. No 594/1999. ${ }^{1}$ Para la directiva 2002/44/CE 3 , en Z se obtuvo un Nivel de Riesgo Crítico en todas las condiciones de evaluación excepto para el caso "con carga" donde el Nivel de Riesgo fue Importante; en Y dio un Nivel de Riesgo Importante para la exposición "sin carga" y "sin carga-con carga"; en X la exposición resultó con Nivel de Riesgo Bajo. Este desempeño se atribuye a que el sistema de suspensión con resortes y amortiguador hidráulico, prácticamente, no reducen vibración en el rango de frecuencia entre $1 \mathrm{~Hz}$ y $25 \mathrm{~Hz}$, reduciendo levemente en torno a la frecuencia de $5 \mathrm{~Hz}$ donde se encuentra la componente de vibración principal que afecta al siento en su base y que es transmitida prácticamente en su totalidad al operador del vehículo. Los valores SEAT en X e Y son superiores al valor 1 . 
Tabla 10.

Evaluación Exposición, "sin carga".

Tipo de Asiento

\begin{tabular}{|c|c|c|}
\hline \multicolumn{3}{|c|}{$\begin{array}{c}\text { Exposición diaria } A(8) \\
\text { con una exposición de } 6 \text { hrs } \\
E A V=0.5 \mathrm{~m} / \mathrm{s}^{\wedge} 2 \\
E L V=1.15 \mathrm{~m} / \mathrm{s}^{\wedge} 2\end{array}$} \\
\hline (8) & Ay(8) & $\operatorname{Ax}($ \\
\hline 92 & 0,43 & 0,3 \\
\hline 61 & 0,45 & \\
\hline 81 & 0,42 & \\
\hline 81 & 0,40 & \\
\hline
\end{tabular}

\begin{tabular}{|c|c|c|c|c|c|}
\hline \multicolumn{3}{|c|}{$\begin{array}{c}\text { VDV, tiempo de medición de } \\
20 \text { min y exp de } 6 \text { hrs. } \\
E A V=9.1 \mathrm{~m} / \mathrm{s}^{\wedge} 1.75 \\
E L V=21 \mathrm{~m} / \mathrm{s}^{\wedge} 1.75\end{array}$} & \multicolumn{2}{|c|}{ Factor de Cresta } & \multirow[b]{2}{*}{$X$} \\
\hline $\mathrm{VDV}_{\mathrm{z}}$ & VDVy & VDVx & Z & $Y$ & \\
\hline 21,41 & 9,58 & 8,32 & 12,43 & 11,54 & 11,65 \\
\hline 33,67 & 9,95 & 9,41 & 8,80 & 10,74 & 7,62 \\
\hline 39,44 & 8,49 & 12,31 & 12,04 & 9,49 & 8,98 \\
\hline 40,46 & 8,01 & 13,00 & 13,77 & 7,41 & 7,50 \\
\hline
\end{tabular}

Tabla 11.

Evaluación Exposición "con carga".

Tipo de Asiento

\section{Exposición diaria A(8) con una exposición de 6 hrs $E A V=0.5 \mathrm{~m} / \mathrm{s}^{\wedge} 2$ $E L V=1.15 \mathrm{~m} / \mathrm{s}^{\wedge} 2$}

Grammer MSG71GBLV

KAB Seating 21/T1

Genérico BF2-3

Genérico BFL-3

\begin{tabular}{c|c|c|}
\hline Az(8) & Ay(8) & Ax(8) \\
\hline 0,51 & 0,39 & 0,34 \\
0,92 & 0,40 & 0,38 \\
0,95 & 0,38 & 0,50 \\
0,92 & 0,32 & 0,49 \\
\hline
\end{tabular}

VDV, tiempo de medición de 10 min y exp de 6 hrs. $\mathrm{EAV}=9.1 \mathrm{~m} / \mathrm{s}^{\wedge} 1.75$ $\mathrm{ELV}=21 \mathrm{~m} / \mathrm{s}^{\wedge} 1.75$

\begin{tabular}{ccc|ccc} 
VDV $_{z}$ & VDV $_{y}$ & VDVx & Z & Y & X \\
12,85 & 8,25 & 7,00 & 10,77 & 8,02 & 7,05 \\
20,44 & 8,27 & 7,65 & 7,35 & 6,10 & 6,39 \\
22,02 & 7,37 & 10,49 & 9,07 & 6,06 & 7,37 \\
21,23 & 6,18 & 9,88 & 10,18 & 6,10 & 7,40
\end{tabular}

Tabla 12.

Evaluación Exposición "sin carga y con carga".

Tipo de Asiento

Exposición diaria $A(8)$
con una exposición de 6 hrs
$E A V=0.5 \mathrm{~m} / \mathrm{s}^{\wedge} 2$
$\mathrm{ELV}=1.15 \mathrm{~m} / \mathrm{s}^{\wedge} 2$

VDV, tiempo de medición de 30 min y exp de 6 hrs. $\mathrm{EAV}=9.1 \mathrm{~m} / \mathrm{s}^{\wedge} 1.75$ $\mathrm{ELV}=21 \mathrm{~m} / \mathrm{s}^{\wedge} 1.75$

\begin{tabular}{l|ccc|} 
& $\operatorname{Az}(8)$ & $\operatorname{Ay}(8)$ & $\operatorname{Ax}(8)$ \\
\hline Grammer MSG71GBLV & 0,87 & 0,43 & 0,39 \\
KAB Seating 21/T1 & 1,51 & 0,45 & 0,45 \\
Genérico BF2-3 & 1,67 & 0,42 & 0,59 \\
Genérico BFL-3 & 1,73 & 0,40 & 0,63 \\
\hline
\end{tabular}

$\mathrm{VDV}_{\mathrm{z}}$

19,60

30,83

35,96

36,69

\begin{tabular}{|c|c|}
\hline VDVy & VDVx \\
\hline 9,19 & 7,93 \\
\hline 9,47 & 8,91 \\
\hline 8,15 & 11,79 \\
\hline 7,43 & 12,03 \\
\hline
\end{tabular}

\begin{tabular}{|c|ccc|}
\hline VDVx & Z & $Y$ & $X$ \\
7,93 & 13,92 & 11,88 & 12,03 \\
8,91 & 9,73 & 10,99 & 7,99 \\
11,79 & 13,49 & 9,80 & 9,38 \\
12,03 & 15,02 & 7,73 & 7,93 \\
\hline
\end{tabular}

Tabla 13.

Evaluación Exposición "Típica", 30\% sin carga-70\% con carga.

\begin{tabular}{|c|c|c|c|c|c|c|c|c|c|}
\hline \multirow[t]{2}{*}{ Tipo de Asiento } & \multicolumn{3}{|c|}{$\begin{array}{c}\text { Exposición diaria } A(8) \\
\text { con una exposición de } 6 \text { hrs } \\
E A V=0.5 \mathrm{~m} / \mathrm{s}^{\wedge} 2 \\
E L V=1.15 \mathrm{~m} / \mathrm{s}^{\wedge} 2\end{array}$} & \multicolumn{3}{|c|}{$\begin{array}{l}\text { VDV, tiempo de medición de } \\
30 \mathrm{~min} \text { y exp de } 6 \mathrm{hrs} \text {. } \\
E A V=9.1 \mathrm{~m} / \mathrm{s}^{\wedge} 1.75 \\
E L V=21 \mathrm{~m} / \mathrm{s}^{\wedge} 1.75\end{array}$} & \multicolumn{2}{|c|}{ Factor de Cresta } & \multirow[b]{2}{*}{$X$} \\
\hline & $\mathrm{Az}(8)$ & $\operatorname{Ay}(8)$ & $\operatorname{Ax}(8)$ & $\mathrm{VDV}_{\mathrm{z}}$ & $\mathrm{VDV}_{\mathrm{y}}$ & VDVx & Z & $Y$ & \\
\hline KAB Seating 21/T1 & 1,17 & 0,42 & 0,40 & 26,69 & 8,88 & 8,30 & 11,96 & 10,01 & 7,76 \\
\hline Genérico BF2-3 & 1,27 & 0,39 & 0,53 & 30,72 & 7,76 & 11,13 & 15,96 & 8,63 & 9,33 \\
\hline Genérico BFL-3 & 1,25 & 0,35 & 0,54 & 31,19 & 6,89 & 11,11 & 18,06 & 8,16 & 8,41 \\
\hline
\end{tabular}

\section{Asiento GENÉRICO BF2-3}

Resultó con un SEAT promedio en el eje Z de 1,01 (RMS) y 1,03 (VDV) que indica una amplificación de 1\% y 3\% en las vibraciones existentes en su base. En el mismo eje, en todas las condiciones de exposición estudiadas, se sobrepasa el límite permisible del D.S. $594 / 1999^{1}$. Para la directiva $2002 / 44 / \mathrm{CE}^{3}$, en Y se obtuvo un Nivel de Riesgo Bajo para todas las condiciones de exposición; en $\mathrm{X}$ las vibraciones en todos los casos resultaron con un Nivel de Riesgo Importante. Este desempeño se atribuye a que la transmisibilidad es cercana al valor 1 en torno a los $5 \mathrm{~Hz}$ y superior al valor 1 hacia las bajas y altas frecuencias, decayendo a partir de los $25 \mathrm{~Hz}$. Los valores SEAT en X e Y son superiores al valor 1.

\section{Asiento GENÉRICO BFL-3}

En el eje Z, resultó un SEAT promedio de 0,82 (RMS) y 0,8 (VDV) que indica una reducción de $18 \%$ y $20 \%$ en las vibraciones. Para el DS No 594/19991, en todas las condiciones de evaluación, la exposición sobrepasó el límite permisible para el eje Z; en los ejes $\mathrm{X}$ e $\mathrm{Y}$ las aceleraciones se encontraron bajo los respectivos límites permisibles. Para la directiva $2002 / 44 / \mathrm{CE}^{3}$, en todas las condiciones de evaluación la exposición obtuvo un Nivel de Riesgo Crítico; en Y, todos los casos resultaron con un Nivel de Riesgo Bajo; en $\mathrm{X}$, todos los casos obtuvieron un Nivel de Riesgo Importante por superarse el límite de acción ocupacional. El desempeño de este asiento se explica por el descenso de la curva de transmisibilidad a un valor cercano a 0,75 en torno a la frecuencia de $5 \mathrm{~Hz}$, donde se ubica la componente vibratoria principal que afecta la base del asiento y que es filtrada hacia el operador del vehículo, aproximadamente en esta proporción. Los valores SEAT en X e Y son superiores al valor 1.

\section{DISCUSIÓN Y RECOMENDACIONES}

En base a los resultados obtenidos, el asiento con suspensión neumática fue superior en la reducción de las vibraciones respecto a los otros seleccionados para este tipo de vehículo, con reducción del orden de 43\%. Aunque presentó factores de cresta altos, 
mantiene un control efectivo de las vibraciones, lo cual se confirma con la evaluación del D.S. $\mathrm{N}^{\circ} 594 / 1999^{1}$, donde la exposición ocasionada por este asiento resultó bajo los límites permisibles y para una condición de alta vibración de entrada (ver Gráfico 1), debido al uso de una superficie de desplazamiento irregular y a la conducción de la grúa de horquilla sin carga, que serían los factores adversos más influyentes en la generación de vibración y exposición de los operadores, según los antecedentes revisados. La tecnología de reducción de este tipo de asiento resultó ser más efectiva en el control de la exposición que la de los asientos convencionales y asiento con suspensión que incorpora un amortiguador hidráulico.

El factor SEAT permitió comprobar que el asiento de la grúa de horquilla constituye un elemento capaz de reducir o amplificar la vibración que expone al operador del vehículo, dependiendo de sus características. En general, en la dirección del eje Z, donde las vibraciones son predominantes, los valores SEAT (VDV) indicaron reducciones desde un 43\% (Grammer MSG71GBLV) hasta un 3\% de amplificación (Genérico BF2-3), diferencias que se atribuyen al diseño, tecnología y elementos utilizados en la elaboración de cada modelo, por lo que un asiento específicamente ajustado para reducir vibración puede constituirse como un elemento efectivo de protección para el operador ante las vibraciones de relevancia ocupacional. Para evaluar la efectividad de un asiento en la reducción de las vibraciones se recomienda que, paralelamente al estudio de la transmisibilidad, obtención del SEAT, se realice un plan de mediciones que permita evaluar la exposición de acuerdo a los límites permisibles que establece el Decreto Supremo 594/19991 u otra referencia normativa.

Se recomienda solicitar al fabricante de los asientos originales y de recambio datos técnicos sobre su capacidad de reducción de vibra- ción, expresados en términos de SEAT o transmisibilidad por banda de frecuencia, dado que con estos datos y el espectro de frecuencia de la vibración del vehículo se puede estimar con una buena aproximación la respuesta que tendría el asiento, una vez instalado, y cuál sería la exposición del trabajador. Adicionalmente, se recomienda no considerar la actual publicidad de asientos antivibración que indican reducciones de hasta un 70\% de la "vibración nociva", lo cual no concuerda con lo encontrado.

Considerando que el modelo de evaluación del D.S.N ${ }^{\circ} 594^{1}$ no incluye factores como las vibraciones con componentes impulsivas y factores de cresta superiores al valor 9, se recomienda utilizar otros modelos de evaluación del riesgo para este tipo de exposiciones, como son los señalados por ISO2631- ${ }^{2}$ y la directiva 2002/44/CE. ${ }^{3}$ También, se recomienda el uso de la norma IS021631-5 del 2004 que propone un modelo de evaluación basado en la predicción del daño en la columna vertebral a partir de mediciones de las vibraciones en los asientos.

Respecto a posibles líneas de aplicación y trabajo a futuro basado en esta investigación, está el ampliar la cantidad de asientos en estudio para disponer de más alternativas en la selección de medidas de control de la vibración. También se puede efectuar un estudio de la efectividad de estos asientos o similares en otro tipo de vehículos, donde la vibración se reconoce como un factor de riesgo para la salud como ocurre en vehículos para el movimiento de tierras, vehiculos de carga y transporte dentro y fuera de carretera, entre otros. Otra posible aplicación sería desarrollar un sistema anexo a un asiento de bajo costo (como los genéricos usados en este estudio), que permita reducir la vibración que afecta al operador, lo que podría efectuarse con una base de soporte y un sistema de aislamiento viscoelástico diseñado apropiadamente para reducir las aceleraciones en el soporte del asiento.
1. Chile. Ministerio de Salud. Decreto Supremo No 594. Reglamento sobre condiciones sanitarias y ambientales básicas en los lugares de trabajo. Santiago: MINSAL; 1999.

2. International Organization for Standardization. ISO 2631-1. Evaluation of human exposure to whole-body vibration. Part. 1: General Requirements. Geneva: International Organization Standardization; 1997.

3. DIRECTIVA 2002/44/CE DEL PARLAMENTO EUROPEO Y DEL CONSEJO. Sobre las disposiciones minimas de seguridad y de salud relativas a la exposición de los trabajadores a los riesgos derivados de los agentes físicos (vibraciones). Madrid: INSHT; 2002.

\section{Referencias complementarias}

1. Drug $C$, Barbu $D$, Lache $S$. Vibration and the human body. Manag Technol Eng. 2007;6 (16):00.

2. Seidel H, Griffin MJ. Vibraciones, Vibraciones de cuerpo completo. En: O.I.T. Enciclopedia de salud y seguridad en el trabajo. Cap. 50. Madrid: Ministerio de Trabajo y Asuntos Sociales; 1998.

3. Griffin MJ. Handbook of human vibration. London: Elsevier; 1996.
4. Viruet HB, Genaidy A, Shell R, Salem S, Karwowski W. Effect of forklift operation on lower back pain: An evidence-based approach. Hum Factors Man. 2008;18:125-151.

5. Malchaire J, Piette A, Mullier L. Vibration exposure on fork-lift trucks. Am Occup Hyg. 1996;40: 79-91.

6. Motmans R. Reducing whole body vibration in forklift drivers. Work. 2012; 41(Suppl.1):2476-2481.

7.- Joubert D. A holistic approach to control of whole-body vibration exposure in forklift drivers. Queensland: Central Queensland University; 2002.

8. Chile. Instituto de Salud Pública. Protocolo para la aplicación del D.S. № 594/99 del MINSAL, Titulo IV, párrafo $3^{\circ}$ Agentes Físicos - Vibraciones. Santiago: ISP; 2012.

9. International Organization for Standardization. ISO 8041: Human-response vibration measuring instrumentation. Geneva: International Organization Standardization (ISO); 2005.

10. KAB SEATING. Vehicles seating: Ergonomically designed Seating for Hardworking Environments [en línea]. Northampton: KAB Seating Ltd.; 2014 [consultado 7 ene 2014]. Disponible en: www.kabseating.com/vehicle/ product.asp? product $=47 \&$ grouptype $=$ range $\&$ rangeid $=11$. 